\title{
Comprehensive Analysis of Augmented Reality Technology in Modern Healthcare System
}

\author{
Jinat Ara $^{1}$ \\ Jahangirnagar University \\ Bangladesh
}

\author{
Faria Benta Karim ${ }^{2}$ \\ University of Asia Pacific \\ Bangladesh
}

\author{
Mohammed Saud A Alsubaie ${ }^{3}$ \\ Taif University \\ Saudi Aarabia
}

\author{
Yeasin Arafat Bhuiyan ${ }^{4}$ \\ Dhaka University \\ Bangladesh
}

\author{
Muhammad Ismail Bhuiyan ${ }^{5}$ \\ Sylhet MAG Osmani Medical College \\ Bangladesh
}

\author{
Salma Begum Bhyan ${ }^{6}$ \\ University of the Sunshine coast \\ Australia
}

\author{
Hanif Bhuiyan ${ }^{7 *}$ \\ Data61, CSIRO and CARRS-Q, \\ Queensland University of Technology, Australia
}

\begin{abstract}
The recent advances of Augmented Reality (AR) in healthcare have shown that technology is a significant part of the current healthcare system. In recent days, augmented reality has proposed numerous intelligent applications in the healthcare domain including, wearable access, telemedicine, remote surgery, diagnosis of medical reports, emergency medicine, etc. These developed augmented healthcare applications aim to improve patient care, increase efficiency, and decrease costs. Therefore, to identify the advances of AR-based healthcare applications, this article puts on an effort to perform an analysis of $\mathbf{4 5}$ peerreviewed journal and conference articles from scholarly databases between 2011 and 2020 . It also addresses concurrent concerns and their relevant future challenges including, user satisfaction, convenient prototypes, service availability, maintenance cost, etc. Despite the development of several AR healthcare applications, there are some untapped potentials regarding secure data transmission, which is an important factor for advancing this cuttingedge technology. Therefore, this paper also analyzes distinct AR security and privacy including, security requirements (i.e., scalability, confidentiality, integrity, resiliency, etc.) and attack terminologies (i.e. sniffing, fabrication, modification, interception, etc.). Based on the security issues, in this paper, we propose an artificial intelligence-based dynamic solution to build an intelligent security model to minimize data security risks. This intelligent model can identify seen and unseen threats in the threat detection layer and thus can protect data during data transmission. In addition, it prevents external attacks in the threat elimination layer using threat reduction mechanisms.
\end{abstract}

Keywords-Augmented Reality (AR); healthcare applications; healthcare challenges; AR-based healthcare security issues; dynamic security solution

\section{INTRODUCTION}

Augmented Reality (AR) stands for the comprehension of modern technology that performs through detection and identification of critical intuition and represents the computergenerated perceptual information (Wikipedia). In recent days, AR is considered one of the prominent advanced technologies for its augmentation prototypes. It enhanced its effectiveness and influenced almost every aspect of life, such as emergency service [2], healthcare management [5][3], industrial implementation, education and training [25][33], design and planning [1][4], and many more.

Therefore, several pieces of research have been undertaken to generate more effective AR prototypes [4] and shown outstanding achievements [5]. According to Urakov et al. (2019) [6], AR enhances the visualization of the 3-dimensional holographic image through sophisticated glasses. Again, Elhariri et al. (2018) suggested that augmented reality is the visualization process of 3-D imagining data [7]. In some other studies conducted by Vavra et al. (2017) [8] and Kim et al. (2017) [9], augmented reality was described as a projection and interaction-based technology to interact and project computergenerated images in a real environment. Current AR-related research has resulted in several advanced prototypes that promote $\mathrm{AR}$ as a prominent technology and call for future research to explore new prototypes and strategies [10][11].

These days, the advancement of AR technology in healthcare has become highly significant with its vast arrays of applications. AR-based healthcare systems are being incorporated with computer vision [6], object detection and identification [18], image processing [10][16], image segmentation [12] and cloud computing technology [14][15]. AR-based healthcare solution has achieved several prominent advancements, such as more secure connectivity and privacy across individual patients [17], more speed while providing quality diagnosis and treatment with more reliability [35]. Besides, wireless services, early diagnosis, real-time monitoring, online consultation, tumor detection, diagnosis specialization, m-health service, and personal assessment are the emerging prototypes of AR-based healthcare solutions, as shown in Fig. 1. Similarly, Medication adherence and rehabilitation treatment have also been enhanced through its utilization. Recently, modern healthcare society introduced an e-health policy to implement prominent augmented devices: AR headset and smart glasses [18]. Therefore, day by day, the popularity of augmented technology in the healthcare system has grown dramatically, and more and more works proposed towards its development.

However, from our empirical observation, it is found that previous review studies have some drawbacks, such as i) a significant number of previous review studies did not present a brief discussion of augmented healthcare applications and their services, ii) few of studies put an effort to provide a comprehensive analysis of the previous works and didn't focus on current limitation of this emerging technology, iii) a majority of them considered only m-health application (medication plan, healthy food guidelines, glucose level monitoring, heartbeat 


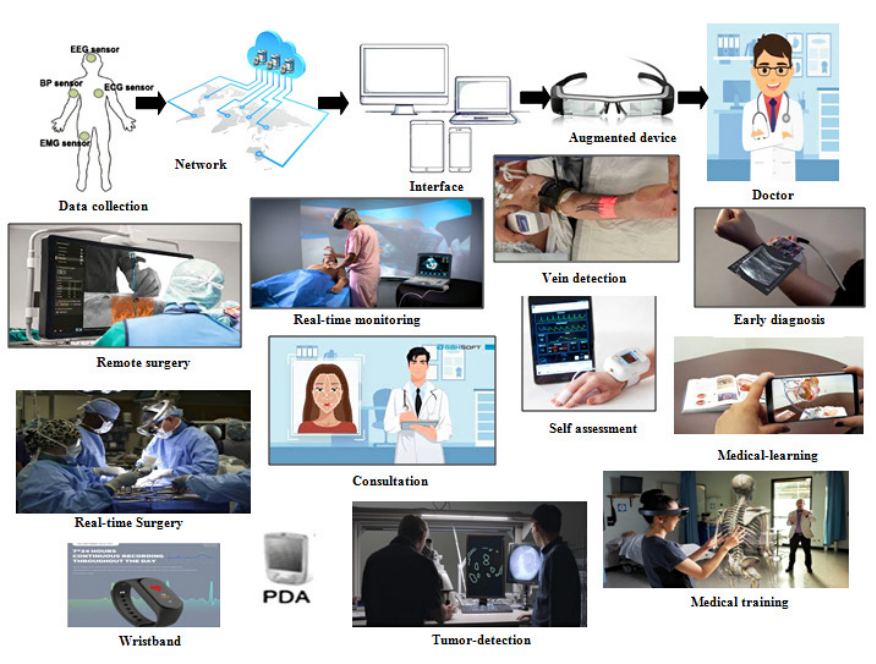

Fig. 1. Current Trends of Augmented Reality in Healthcare Platform.

calculation, etc.) for review/analysis rather than focusing on clinical applications (examines the abnormal joint function, tumor location detection, vain detection, diagnose breast cancer, etc.), and iv) some of the papers addressed recent challenges of augmented healthcare application in short and did not address the possible attacks with proper solutions to mitigate these challenges. Therefore, based on our scientific observation and the author's best knowledge, it can be stated that a comprehensive analysis would be beneficial to identify the limitation and challenges of AR-based healthcare applications. Moreover, this analysis may help to find the solution to these challenges as well as for the further improvement of current applications. This paper aims to investigate the impacts of augmented reality in the healthcare system by addressing above mentioned issues. A brief discussion on augmented healthcare applications (m-health and clinical applications) with their services in the healthcare system is present in this study. Also, a wide range of challenges of the augmented platform in the healthcare system has been demonstrated here with a possible solution against future attacks. Moreover, this paper can be influential in having insights into existing augmented-based healthcare applications. For a further study of AR healthcare solutions, interested readers referred to [23][24][25].

This article has organized as follows: Paper collection and selection for review described in Section 2. Section 3 demonstrates through healthcare applications development prototypes, several augmented mobiles and clinical healthcare applications with services, and current challenges. Section 4 summed up our contribution by addressing several security issues with our proposed intelligent security architecture. Finally, the article concluded with a conclusion and a recommended direction for future research.

\section{Resources of Related Papers}

\section{A. Paper Selection Process}

To find the published research articles, we have considered five popular databases in the computer science and health informatics domain for recent development and updated approaches (shown in Fig. 2).

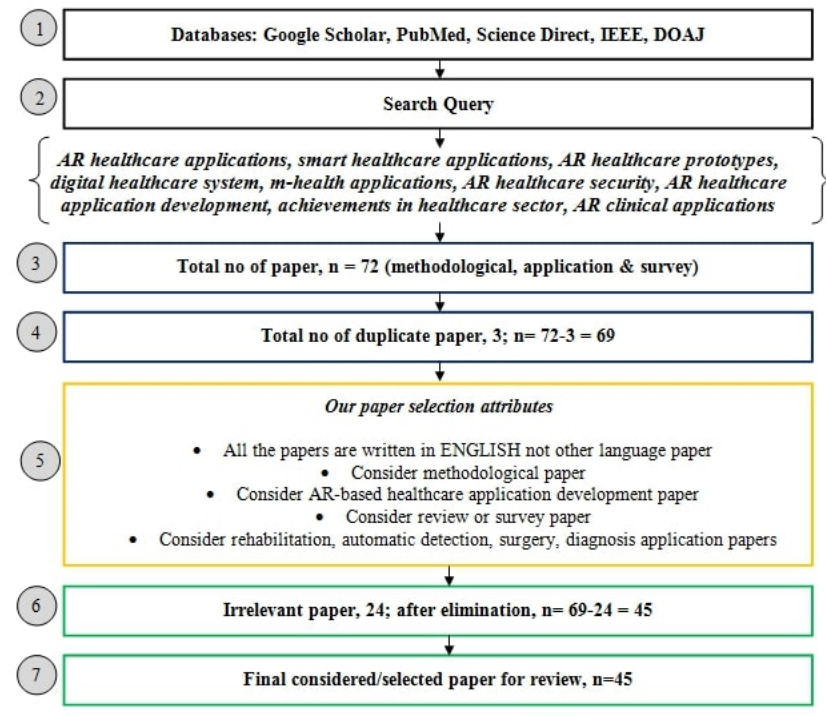

Fig. 2. Related Article Collection and Selection Through Search Query and Predefined Criteria using PRISMA Flow Diagram.

We have considered search queries for searching the relevant papers from the selected database according to the relevancy of our work. For this work, we investigated both peer-reviewed journal and conference articles from 2011 to 2020. Primarily, 72 related articles were found relevant, and after further evaluation, 27 articles have excluded, and 45 articles have finally selected according to our aim. The paper selection process performs following the PRISMA approach. Fig. 2 shows the article extraction and selection process using the PRISMA flow diagram. In Fig. 2, at stage-1, to extract recent publications of augmented and healthcare technology, we considered IEEE, Google Scholar, PubMed, Science Direct, and Directory of open access journal (DOAJ) databases. At stage-2, article searching performs through several keywords such as Augmented Reality (AR) healthcare application, Digital healthcare system, AR-based smart-healthcare applications, AR healthcare application developments, AR-based m-health, recent AR achievements in the health sector, AR healthcare Security, AR clinical application, etc. We added the advanced search option to reveal the most recent articles of our intended domain. Regarding search keywords, we have extracted 72 papers that belong to the methodological approaches, real-life AR healthcare applications development, comparative analysis, survey, and review papers (at stage-3). We performed a manual checking to identify duplicates papers. Three papers have been identified as duplicate and eliminated (stage-4). At stage-5, we set our selection attributes to evaluate the collected papers according to their relevancy with our study aim. Those articles that did not match our selection criteria (Fig. 1, stage-5) did not consider for review. Mostly, those papers that are not written in English and not directly related to the healthcare domain have been marked irrelevant and excluded. After evaluating, 24 articles were removed from the total number (stage-6), and 45 papers were selected for final review (stage-7).

\section{B. Papers Statistics Information}

Here, we have categorized the previous articles according to their year of publication, publication database, proposed 
TABle I. CATEgorical OVerview of Selected Papers for Review

\begin{tabular}{|c|c|c|c|c|c|c|c|}
\hline No. & Authors & Country & Publication year & Published database & Category & Journal & Conference \\
\hline 1 & White et. al. [1] & Ireland & 2018 & Google Scholar & Methodological & - & Yes \\
\hline 2 & Jeong et al. [2] & South Korea & 2017 & DOAJ & Review & Yes & - \\
\hline 3 & Agrawal et al. [3] & India & 2018 & IEEE & Application Development & - & Yes \\
\hline 4 & Riva et al. [4] & Italy & 2016 & DOAJ & Comparative Analysis & Yes & - \\
\hline 5 & Sutherland et al. [5] & Canada & 2019 & Google Scholar & Review & Yes & - \\
\hline 6 & Urakov et al. [6] & USA & 2019 & Science Direct & Application Development & Yes & - \\
\hline 7 & El-Hariri et al. [7] & Canada & 2018 & DOAJ & Methodological & Yes & - \\
\hline 8 & Vávra et al. [8] & Czech Republic & 2017 & PubMed & Review & Yes & - \\
\hline 10 & Frajhof et al. [10] & Brazil & 2018 & DOAJ & Methodological & Yes & - \\
\hline 11 & Mahmood et al. [11] & UK & 2018 & Science Direct & Comparative Analysis & Yes & - \\
\hline 12 & Wilhelm et al. [12] & Germany & 2018 & PubMed & Review & Yes & - \\
\hline 13 & Liu et al. [13] & France & 2018 & IEEE & Comparative Analysis & - & Yes \\
\hline 14 & Jones et al. [14] & USA & 2018 & DOAJ & Comparative Analysis & Yes & - \\
\hline 15 & Chen et al. [15] & China & 2011 & IEEE & Methodological & - & Yes \\
\hline 16 & Ahad et al. [16] & Bangladesh & 2018 & PubMed & Comparative Analysis & Yes & - \\
\hline 17 & Pereira et al. [17] & Chile & 2019 & Science Direct & Methodological & Yes & - \\
\hline 20 & Viglialoro et al. [20] & Italy & 2019 & DOAJ & Review & Yes & - \\
\hline 21 & Garrett et al. [21] & Canada & 2018 & DOAJ & Comparative Analysis & Yes & - \\
\hline 22 & Wong et al. [22] & USA & 2018 & PubMed & Review & Yes & - \\
\hline 23 & Yoon et al. [23] & USA & 2018 & Google Scholar & Review & Yes & - \\
\hline 24 & Belmustakov et al. [24] & USA & 2018 & Science Direct & Review & Yes & - \\
\hline 25 & Uppot et al. [25] & USA & 2019 & PubMed & Review & Yes & - \\
\hline 26 & Rabbi et al. [26] & Pakistan & 2012 & Google Scholar & Review & Yes & - \\
\hline 27 & Pflugi et al. [27] & Switzerland & 2017 & Google Scholar & Application Development & - & Yes \\
\hline 28 & García-Cruz et al. [28] & Spain & 2018 & Science Direct & Comparative Analysis & Yes & - \\
\hline 29 & Nakamoto et al. [29] & USA & 2012 & DOAJ & Review & Yes & - \\
\hline 30 & Daher et al. [30] & USA & 2017 & IEEE & Comparative Analysis & - & Yes \\
\hline 31 & Hemanth et al. [31] & Brazil & 2020 & Google Scholar & Comparative Analysis & Yes & - \\
\hline 32 & Moro et al. [32] & Australia & 2017 & Google Scholar & Comparative Analysis & Yes & - \\
\hline 33 & Lahanas et al. [33] & Greece & 2016 & DOAJ & Survey & Yes & - \\
\hline 34 & Maier-Hein et al. [34] & Germany & 2013 & Science Direct & Methodological & Yes & - \\
\hline 35 & Chen et al. [35] & USA & 2018 & Google Scholar & Application Development & - & Yes \\
\hline 36 & Tabrizi et al. [36] & Germany & 2015 & Google Scholar & Application Development & Yes & - \\
\hline 39 & Monge et al. [39] & Portugal & 2018 & IEEE & Methodological & - & Yes \\
\hline 40 & Hsiao et al. [40] & Taiwan & 2015 & Google Scholar & Methodological & Yes & - \\
\hline 41 & Shinomiya et al. [41] & Japan & 2018 & Science Direct & Review & Yes & - \\
\hline 42 & Gallos et al. [42] & Greece & 2018 & PubMed & Survey & Yes & - \\
\hline 43 & Ingeson et al. [43] & Sweden & 2018 & Google Scholar & Methodological & Yes & - \\
\hline 44 & Chamberlain et al. [44] & USA & 2016 & PubMed & Methodological & - & Yes \\
\hline 45 & Hayhurst et al. [45] & England & 2018 & Google Scholar & Review & Yes & - \\
\hline
\end{tabular}

category, journal paper, and conference paper. The total number of 45 articles have summarized in Table 1 . These articles have been selected based on some predetermined criteria (Fig. 2). From Table 1, the following observation may conclude. First, from 2018 to the present, AR healthcare application has become a serious concern for most the computer scientist and healthcare professionals. Second, the number of comparative analysis and review of AR healthcare applications have dramatically increased in recent days. In contrast, the number of application development articles has decreased due to challenges for ensuring appropriate services and security issues. The detailed statistics of selected articles have shown in the following sections.

1) Study year and reference database: Here, we conclude the statistics of the considered articles according to their publication year (2011-2020) and referencing databases (PubMed, Science Direct, DOAJ, Google Scholar, and IEEE), which shows in Fig. 3. Among 45 articles, a significant number of works have published in 2018, 2017, and 2016.

Among five databases, PubMed published ten articles, Science Direct published seven articles, DOAJ published nine articles,

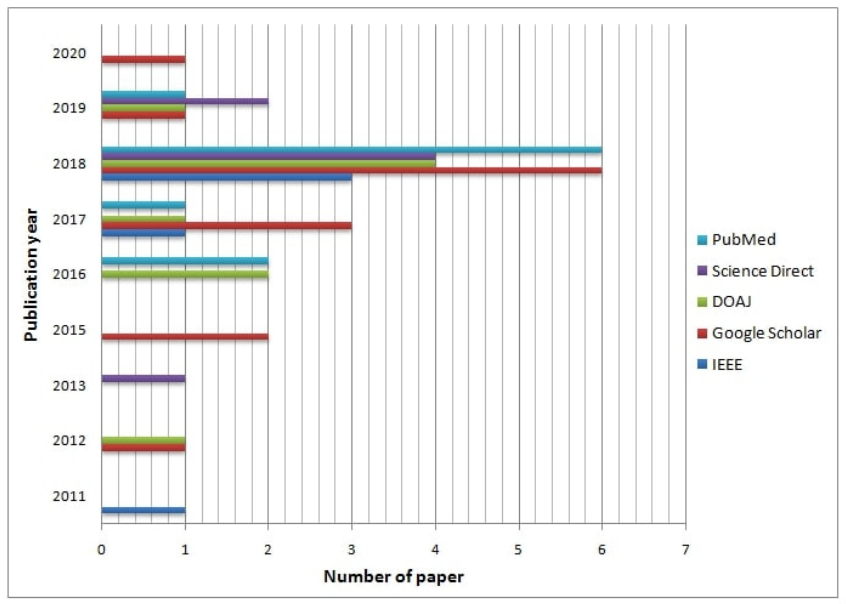

Fig. 3. Number of Articles According to their Publication Year and Referencing Databases. 
Google Scholar published thirteen articles, and IEEE published five articles. Moreover, a maximum number of the article we have found for the year 2018. Besides, Google Scholar $\&$ PubMed is the more popular database or resource for augmented healthcare domains.

2) Category of study articles: To understand the effectiveness of previous works, we analyzed our selected fortyfive articles into five categories: methodological, application development, comparative analysis, review, and survey paper. At the same time, we classified these studies into journals and conference publications. Fig. 4 shows that among a total of 45 articles, review, comparative analysis, and methodological papers are maximum in consideration. Among the explained studies, the proposal for methodological studies is ten, six for application developments, eleven for comparative analysis, fifteen for review paper, and three for the survey. The majority of these journal and conference proceedings come from healthcare engineering, computer science, biomedical engineering, and bioinformatics.

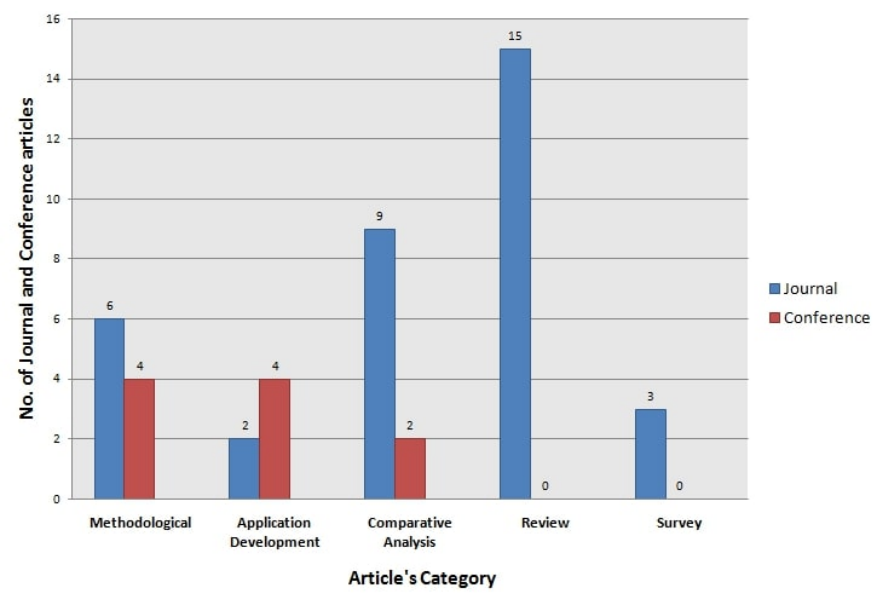

Fig. 4. Number of Articles in Each Category with Paper Status.

3) Country of study articles: In this study, we considered articles from both developing and developed countries. Figure 5 shows the number of articles according to their country where majority from the USA, Canada, Germany and Brazil and few from UK, South Korea, Greece, Italy, and France. Compared to these countries Australia, India, Bangladesh, Pakistan, and others show less interest in AR-based healthcare research.

\section{Comprehensive AnAlysis of Selected ARticles}

After article selection (explain in Section 2), our primary objective is to perform a comprehensive analysis of these 45 selected articles. To do our analysis, here we have considered our research questions regarding how augmented technology facilitated in healthcare domain; identify the recent augmented healthcare applications with services, and current challenges of existing augmented healthcare applications. After exploring these aspects, based on our observation, we proposed a model to mitigate the recent augmented healthcare application's limitation (Section IV-C).

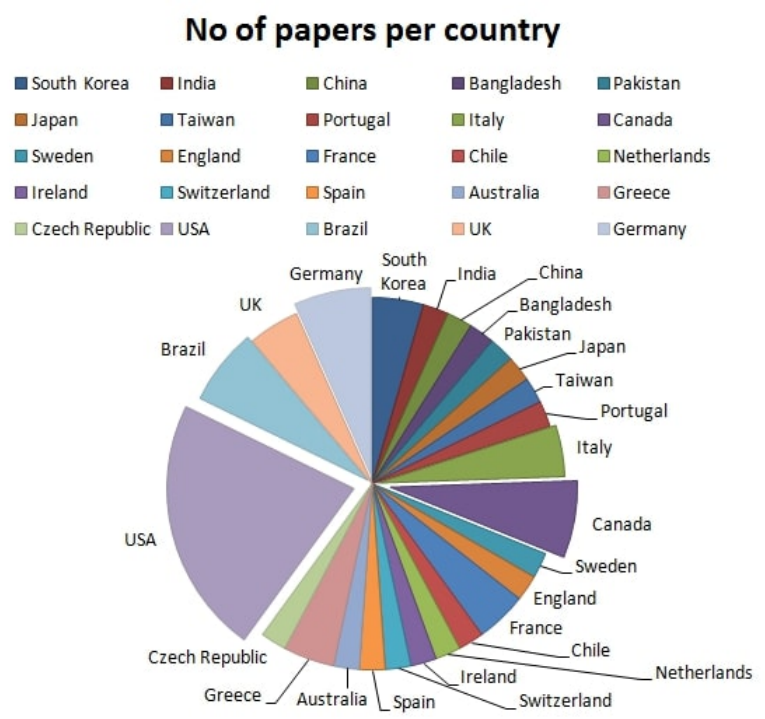

Fig. 5. Number of Articles Per Country.

\section{A. Augmented Reality in Healthcare System}

Nowadays, AR technology has attracted significant attention to developing applications that healthcare professionals and patients directly use. Augmented healthcare solutions may apply to a diverse array of fields, including diagnosis, surgery, rehabilitation, monitoring, guidance, education, etc. Generally, AR technology facilitated the healthcare system through its numerous services and platform [29]. The AR service platform refers to a framework that focuses on information acquisition, proper data organization; ensuring data support and providing consistent services, as shown in Fig. 6.

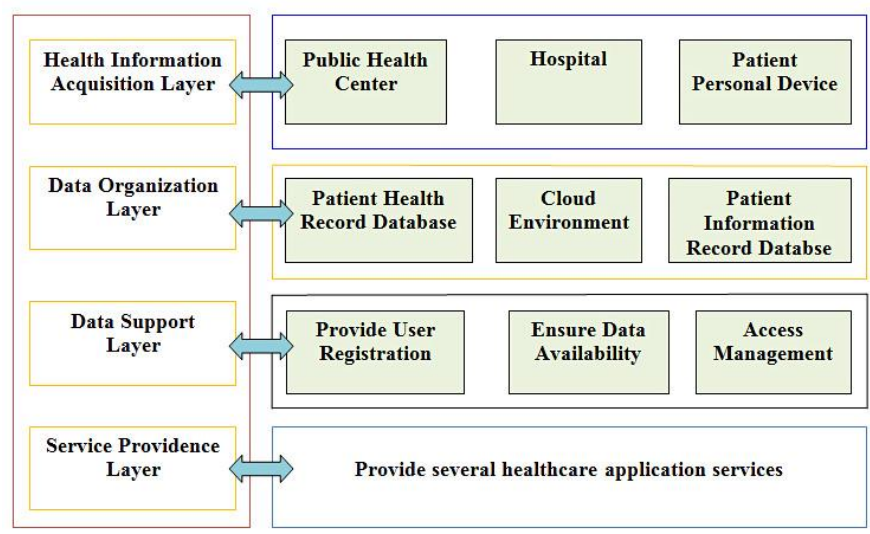

Fig. 6. The Process of Augmented Healthcare Application Services.

1) Health information acquisition layer: It refers to collecting healthcare information or patient health reports shared by patients or public health centers or hospitals to make available patient health records to medical specialists or doctors. Generally, healthcare or hospital authority collects these health data and stores them on the hospital database.

2) Data organization layer: The data organization layer is the primary layer of the AR-based healthcare application 
development process. It benefits transferring real-world information into a virtual environment. This layer extracts the health information from the healthcare database and stores it into a cloud environment to support data preprocessing and model creation. Cloud environment or cloud server refers to data (health data) in the server [27]. Cloud databases provide services for healthcare applications during data examination or visualization. The data preprocessing performed to reduce the noise in MRI and X-ray data. Finally, model creation helps create a visual object for the effective organization of 3-D data [26]. This process allows the generation of a 3-D model from 2-D or 3-D health images or data.

3) Data support layer: The data support layer provides services through user registration, data availability, and accessing opportunities. To reduce unknown threats during communication it prerequisites the registration panel. Based on authenticity confirmation, it ensures data availability and accordingly grants access. The authentication process of numerous prototypes ensures through the network interface. Network interface provides the network layer to allow secure communication of the entire prototype of AR healthcare application. Network Layer ensures security through network platforms and network protocols. A network platform is a medium that generates frequencies for communication. Third-generation technology (3G) and fourth-generation technology (4G) are the most popular network platform or network technologies for AR-based healthcare applications. Managing data and ensuring secure connectivity are the prior responsibilities of network protocols. Two widely used protocols are User Datagram Protocol (UDP) and Transmission Control Protocol (TCP). UDP and TCP are responsible for data transmission where UDP is connectionless, reliable, and TCP, errorless.

4) Service providence layer: The service providence layer refers to the user interface that allows the extraction of virtual environment data according to availability and accessibility. This layer is also called the process of data collaboration. It helps healthcare specialists overlay the virtually created imagining model on real-time patients' bodies using AR devices. During surgery or diagnosis, on-demand health data accessing is the prime objective of this interface. The majority of healthcare solutions are using smart displays or glass. To detect an object, image, or text projection, Head Mounted Display and Holographic Display device are significant. In recent days, HMD has implemented various healthcare solutions like surgery, diagnosis, assessment, etc. Some prominent functionality of it includes recognizing voice comments, tracking eye movements and gestures movements. At present, powerful and prototype-based HMD devices are OHMDs and OST-HMD. Similarly, a Holographic display is a lighting technology that visualizes a three-dimensional image of objects with better accuracy. Among several holographic displays, HoloLens and Oculus rift is the most used one in healthcare applications. In contrast, smart glass is a wearable, hands-free control system that collects patient's physical information and provides accurate solutions during surgery and diagnosis. It helps to take pictures, record videos, recognize voice instruction, and lessen the requirement of looking on a different screen while examining the medical test report [28]. Smart glasses are being categorized as optical glass and video glass for this study. It is worth mentioning that the majority of AR-based healthcare application uses optical glasses for its augmented functionality.
The most prominent optical glasses are VuFine and Google Glass. Video glasses are called personal media viewers as they provide hands-free convenience. These are categorized into Vuzix, Epson Moverio and Atheer air.

\section{B. AR Healthcare Applications and Services}

According to our observation regarding the context of 45 healthcare articles, no standard definition of AR healthcare services has been found. As a result, it gets hard to categorize the healthcare application and their services. However, here, we classified augmented healthcare applications into six categories: including Diagnosis, Surgery, Monitoring, Guidance, Rehabilitation, and Medical Education. We have summarized the available AR healthcare applications and their services as following.

1) Diagnosis Applications: Eye Diagnosis, Cancer Diagnosis, Cardiac Diagnosis, Tumor Detection, Vein Detection, and Lung Diagnosis [34] [19] [35] [31] [3] [36].

\section{a) Services:}

- Identifies the patient's exact eye diseases: Glaucoma, dry eye condition, etc.

- Using a digital contact lens measures blood sugar levels through a multi-sensor for retinal implanted people.

- Used to diagnose breast cancer in sentinel lymph nodes and prostate cancer in prostatectomy specimens.

- To understand cardiac inner and deeper structure.

- Analyzes the heart condition according to normal, murmur and extra-systolic sound.

- Cardiac data examination, prediction and cardiac arrhythmia treatment.

- Identifying skin incision, skull craniotomy and tumor location.

- Provides feedback on lung conditions and is used to diagnose respiratory disease.

- M-application use for vein detection.

2) Surgery Applications: Orthopedic Surgery, Choledochoscopy Surgery, Dental Surgery, craniofacial Surgery, HIP Surgery, Urology Surgery, Laparoscopy Surgery [22] [29] [38] [41].

\section{a) Services:}

- Helps orthopedic surgeon to examine abnormal joint function.

- Identifies the bone structure through 3D computed tomography (CT) data for orthopedic surgery.

- Identify skin incision, skull craniotomy and tumor location for Choledochoscopy surgery.

- Prevents damaging tissues, blood vessels and dental nerves during dental surgery.

- Helps in Endoscopic endonasal transsphenoidal surgery. 
- Used for craniofacial surgery applied in treating orbital hypertelorism, hemifacial microsomia, mandibular angle split osteotomy related abnormalities.

- Use for safely HIP placement.

- Enhances urology surgical procedures.

- Provides automatic relevant information to help dental surgeons.

- Used in stone extraction and biopsy procedure.

- Used for treating orbital hypertelorism, hemifacial microsomia, mandibular angle split osteotomy related abnormalities.

- Used for laparoscopy surgery like endophytic tumor surgery.

- Identifies the bone structure through 3D computed tomography (CT) data.

- $\quad$ Reduces blood loss and risk of chronic renal insufficiency.

3) Monitoring Applications: Glucose Level Monitoring [42], Insulin Dosages Monitoring [43]

\section{a) Services:}

- Continuously monitors glucose level, insulin dosages and suggests appropriate foods.

- Helpful for real-time health monitoring and recommending appropriate specialist.

- Automatically connects to the emergency number to seek help or to find defibrillators.

- Measures blood sugar levels through multi-sensor for retinal implanted people.

4) Guidance Applications: Grocery Shopping, Medication Plan, Smoking Consequence, Exercise Plan, Medicine Scheduling, Hearing Impaired Guidance [44] [45].

\section{a) Services:}

- $\quad$ Provides exact guidelines about healthy food.

- Provides information about allergic food, low-fat diets, and general caloric intake.

- Support for medication plan and medication restrictions.

- Helps hearing impaired people for museum visits.

- Shows the consequence of smoking and stimulates the side effects.

- Shows medicine functionality and using procedure.

- $\quad$ Spreads awareness about exercise and make people fit and healthy.

- Recognizes speech commands and provides information on medical needs.

- Provides exact information about drug names and dosages.
5) Rehabilitation Applications: Smart Physical Rehabilitation [20] [37][39] [40]

\section{a) Services:}

- Provides inpatients and outpatient rehabilitation facilities for musculoskeletal, neurological, r-hematological and cardiovascular systems.

6) Medical Education Applications: Training, 4DVisualization [21] [32] [33].

\section{a) Services:}

- Represents the human organs into 4D anatomies without dissection.

- Helps dental students with both theoretical and practical training.

\section{Current Challenges of AR Healthcare Applications}

One primal focus of this research is to identify the current challenges of existing AR healthcare applications. Also, we noticed several possible future challenges related to augmented healthcare applications. According to the perspective of our considered articles, here we have conceded eight challenges. Several other challenges might exist in the current AR healthcare application that is beyond our scope of this review.

1) Challenges with Data security: Ensure healthcare data security is the most significant aspect of AR healthcare applications. Surprisingly, several exiting applications have no standard security protocols and recently proposed approaches also have a poor security concern. To ensure data security, the implementation of network connection encryption is essential. Providing a complete encrypted or protected data transferring environment for healthcare applications is difficult but adequately needed.

2) Challenges with Specialized platform: The majority of augmented healthcare applications have no standard platform and lack appropriate guidelines. The incompetency to provide authentic and accurate services makes it more insecure and complex. Launching a standard and specialized platform that refers to applications package interface (APIs), framework, and appropriate libraries are challenging for modern healthcare platforms. Failure to ensuring a specialized platform may hamper in manages of code, classes, and documentation also.

3) Challenges with Limited services: From 2011 to the present, several applications have developed for a limited number of healthcare aspects, for example, diagnosis, treatment, assessment, etc. Yet, more healthcare branches should disclose to provide an effective solution, for example, cardiac surgery, Hematopoietic stem-cell transplantation, etc.

4) Challenges with Healthcare information accessing interoperability: The Healthcare Leadership Council (HLC) published several guidelines related to healthcare IT interoperability. For accessing and sharing information, ensuring HLC guidelines are a big concern. 
5) Challenges with Scope of adaptability: Complex functionality and lack of user guidance make a barrier to healthcare application adaptability. Generally, augmented healthcare application is the reflection of integrated digital components. These integrated digital components might introduce some technical barriers, especially for the healthcare specialist and the end-user. Overcoming the technical obstacle is one of the utmost challenges.

6) Challenges with Lack of availability: Several AR healthcare applications launch in the last few years. But a majority of them have been used in the developed country only. High configuration prototypes, training requirements, and cost issues are responsible for these applications limitation in underprivileged countries. Ensuring these applications available for all the country is a challenging issue.

7) Challenges with Implementation cost: Furthermore, the development and implementation cost of the AR healthcare device is another vital issue. Due to the high development cost, several healthcare providers disagree to reconstruct their healthcare platform. Therefore, an increased range of implementation costs raises the barrier to introducing modern and digital technology into healthcare solutions.

8) Challenges with Healthcare device acceptability: The current augmented platform is committed to providing a userfriendly environment with fluent functionality. But sometimes, issues associated with improving consistency and reducing errors can be more viable. Overcoming this challenge may help to increase the device's acceptability. Developing an application with effective functionalities is a relatively focusable issue for AR technology.

9) Challenges with Inappropriate prototypes: Due to inappropriate prototypes, sometimes, unexpected abnormalities such as false recognition, lack of semantic knowledge reduce the effectiveness of augmented healthcare applications. Ensure appropriate prototypes are one of the distinct challenges of healthcare application.

10Challenges with Service availability: Instead of numerous AR applications, providing constant services or longduration services such as surgery visualization, projection, and interaction for as long as 9-12 hours in a continuous manner is relatively challenging.

11)Challenges with Real-time geometry awareness: Realtime geometry awareness is another major issue for researchers and developers. It associates with the process of annotation, tracking location, object detection, and appropriate measurement. Surprisingly, most abnormalities arise during geometry transformation in the augmented application. Therefore, awareness regarding real-time geometry can be a challenging issue for AR platforms.

12)Challenges with Limited research: According to the research statistics, it has shown that AR research on the healthcare domain is insufficient due to limited scope, less popularity for healthcare professionals, ambiguous knowledge, and limited resources. Therefore, most healthcare researchers have not shown their interest to research in the healthcare domain, specifically on augmented platforms.

\section{OUR CONTRIBUTION}

Section 3 shows that several challenges have existed in the recent augmented healthcare platform. Among several current AR healthcare application issues, ensuring data security is one of the major concerns. Generally, augmented healthcare application provides services through a physical layer with overlaid computer-generated information [30]. Therefore, it is not free from external threats, and cybersecurity risk might introduce on the physical layer. AR healthcare applications should address these external threats and common security vulnerabilities during the development phase. Therefore in this section, we addressed several security requirements and attack terminologies to ensure data security and added a security model that might help prevent threats or network attacks during data transmission.

\section{A. Security Requirements}

Security requirements of augmented healthcare applications refer to the property that protects the application platform from unexpected attacks or threats. Figure 7 shows some required security requirements that should focus during application development to ensure application effectiveness and modality.

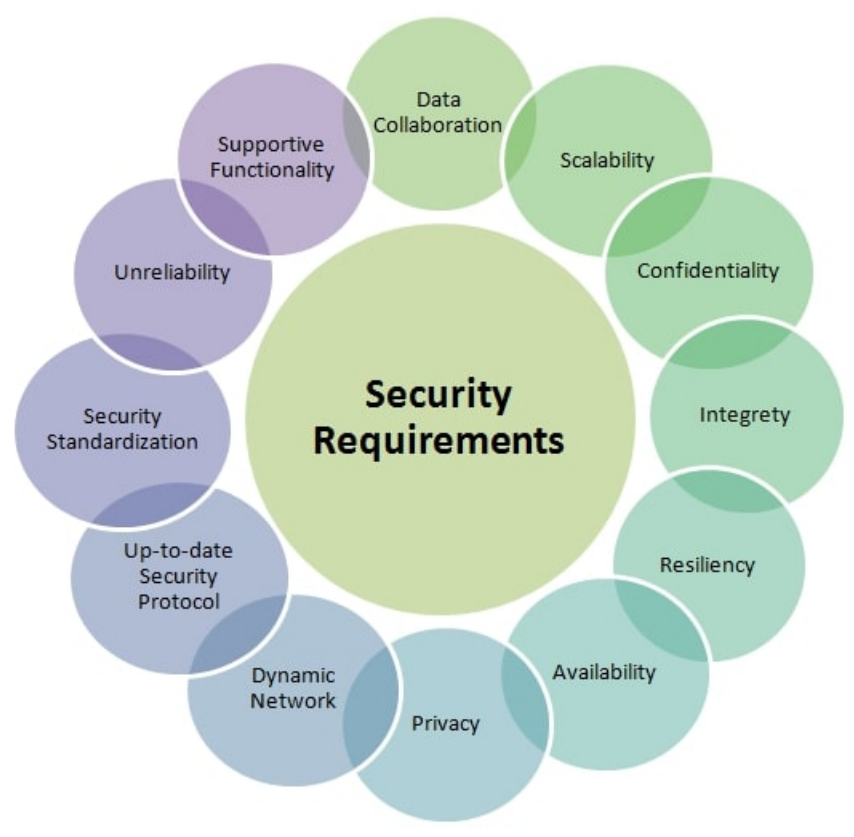

Fig. 7. Security Requirements of AR Platform.

For example, during the collaboration of virtual objects and real-time data, an intruder may attack. The augmented platform should provide security during virtual and real-time object collaboration. At present, the number of augmented healthcare devices has increased and started to connect with the network. Therefore, the augmented platform should ensure confidentiality to prevent unregistered access to medical information to provide medical data security. However, the assorted number of things can modify data while accessing or transmitting. Healthcare solutions should introduce integrity to ensure 
medical information or health data security. Another crucial requirement is resiliency to provide a protective environment if health devices are compromised.

Another major security issue of current healthcare applications is availability. It ensures that data are stored and available in a secure environment and used by the registered authority under secure networks. To increase efficiency and protect patient data from untraceable attacks, ensuring the tracking functionality of all the activities with an authorized detector is essential. Most of the time, lack of awareness about DoS attacks can create opportunities for malicious attackers to hamper data safety while transmitting. Therefore, security awareness should consider sincerely. As we previously mentioned, augmented healthcare devices are wearable technologies to connect to the network anywhere. Hence, deriving a security model for dynamic network topology is highly required.

Designing a dynamic security protocol could increase the security of augmented applications. Surprisingly, augmented Reality Markup Language (ARML) has a lack of security control policy. Lack of security issues and Limited standardization makes it vulnerable, which in turn can introduce security vulnerabilities. In recent days, augmented reality uses computer-aided objects provided by third-party vendors that pave the way for threats such as spoofing, sniffing, data manipulation, and man-in-middle attacks. The AR platform should be aware of these threats and be sure of the authenticity of the generated content during the transmission process to make the application reliable. As augmented healthcare application often uses various unprotected web browsers, it can lead to security breach too.

\section{B. External Attack Terminology}

The augmented platform is not free from external attacks. Attackers may introduce several security threats and affect existing and future augmented healthcare applications, devices, and networks. Sometimes these threats are predictable, while some are hard to detect or predict. In this section, we are going to mention several external threats that reduce application and network effectiveness. Fig. 8 shows some attack terminologies that can make systems vulnerable and reduce effectiveness.

For example, by data-stealing or spreading malicious codes during data transmission or communication, intruders may replace original data or make it unreliable. Through unauthorized access, attackers may modify the data and create confusion and reduce network performance. Also, false information may get injected into the network and minimize data reliability, affect the hardware platform by altering program code, information, or reprogramming. Sometimes an attack can be introduced through software platforms such as operating systems or software applications and take advantage of software vulnerabilities that cause buffering, resource destruction or loss.

\section{Proposed Data Security Model}

Augmented reality-based developed healthcare applications and services are not yet robust but continuing to develop. Therefore, summing up the entire possible vulnerabilities, threats, and attacks associated with augmented medical solutions is difficult. With the expansion of healthcare devices, networks, and applications, several unknown or unseen threats

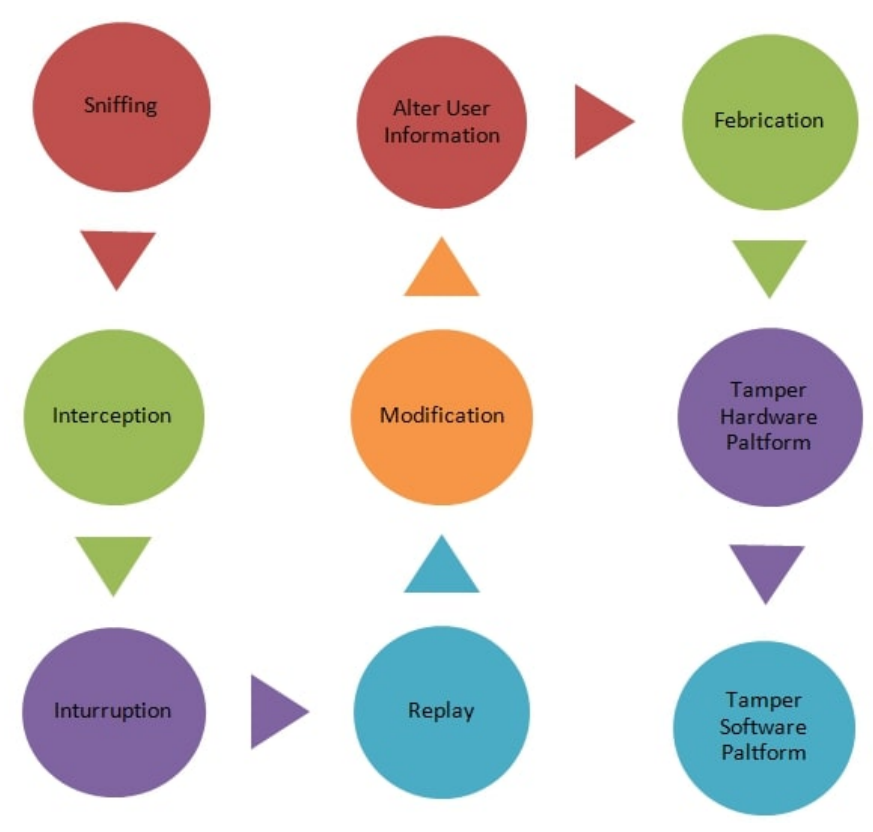

Fig. 8. Possible Attacks for Security Threats.

may initiate during communication or data transmission, or data storage in the cloud.

A dynamic security protocol is essential to mitigate unseen data threats and provide data transmission security. Therefore, security services should design with dynamic properties. An artificial intelligence-based dynamic mechanism might be capable of identifying these unseen attacks. Here we proposed a security model for AR-based healthcare solutions. The proposed model operates through knowledge-based services. Figure 9 presents the proposed scheme that follows three security layers: threat detection, threat reduction, and data protection.

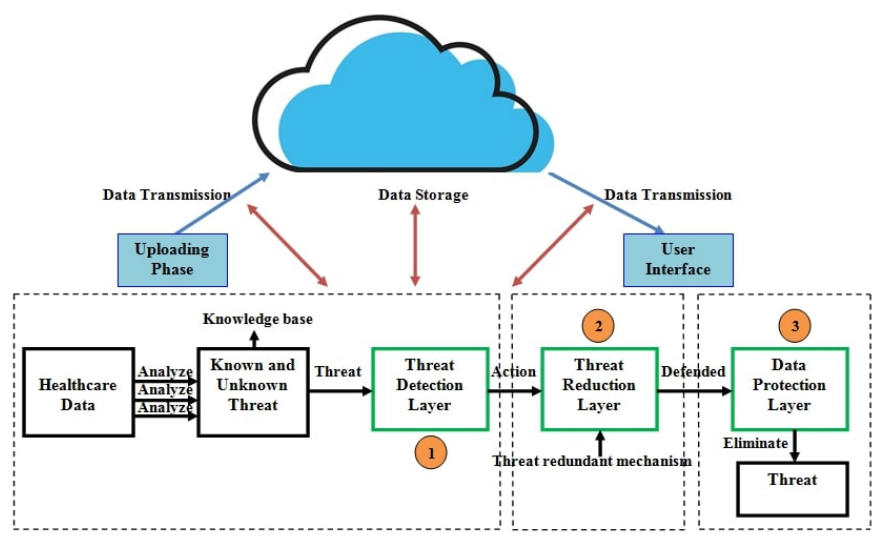

Fig. 9. Proposed Intelligent Security Model.

The threat detection layer is designed to receive healthcare data from healthcare devices and networks and analyzes captured information to identify security threats. Then, to reduce the attack during data transmission, a threat reduction layer is designed. The data protection layer helps protect data by 
defending all identified attacks. This security layer performs through dynamic algorithms and provides a shield against attacks. Upon detection, the threat detection layer issues action commands and shares detection experience with the threat reduction layer. The threat reduction layer defends threats using a redundant threat mechanism. Finally, the data protection layer eliminates detected threats and protects valuable data.

\section{CONCLUSION}

Researchers are vigorously working to invent better technological solutions to enhance the modern health care system throughout the world. The motive of these inventions is to bring a dramatic change in the healthcare sector and reduce the existing complexities. In this paper, we have provided a brief analysis of various AR-based healthcare services and applications. A broad view of the current development strategies regarding present healthcare application architecture and health data processing and accessing procedures have been layout. The article, to some extent, tried to facilitate further development by pointing out several uncovered issues regarding concurrent security requirements and future challenges. The discussion performed in this article on standardization, data availability, service quality, and data protection may help in several ways for future research on AR-based healthcare applications and services. Moreover, this paper also illustrated the importance of augmented reality-based healthcare applications, backed up with present-day market data, which may increase the contribution of several stakeholders for further development.

\section{REFERENCES}

[1] White. G, Cabrera. C, Palade. A, and Clarke. S, Augmented reality in iot, In International Conference on Service-Oriented Computing, pp. 149-160, Springer, Cham, https://doi.org/10.1007/9783- 030-176426_13, 2018.

[2] Jeong. B, and Yoon. J, Competitive intelligence analysis of augmented reality technology using patent information, Sustainability, 9(4),pp. 497, https://doi.org/10.3390/su9040497, 2017.

[3] Agrawal. D, Mane. S. B, Pacharne. A, and Tiwari. S, IoT Based Augmented Reality System of Human Heart: An Android Application, In 2018 2nd International Conference on Trends in Electronics and Informatics (ICOEI), pp. 899-902, IEEE.https://doi.org/10.1109/ICOEI.2018.8553807, 2018.

[4] Riva. G, Baños. R. M, Botella. C, Mantovani. F, and Gaggioli. A, Transforming experience: the potential of augmented reality and virtual reality for enhancing personal and clinical change, Frontiers in psychiatry, 7 , 164, https://doi.org/10.3389/fpsyt.2016.00164, 2016.

[5] Sutherland. J, Belec. J, Sheikh. A, Chepelev. L, Althobaity. W, Chow. B. $\mathrm{J}$, and La Russa. D. J, Applying modern virtual and augmented reality technologies to medical images and models, Journal of digital imaging, 32(1), pp. 38-53, https://doi.org/10.1007/s10278-018-0122-7, 2019.

[6] Urakov. T. M, Wang. M. Y, and Levi. A. D, Workflow caveats in augmented reality-assisted pedicle instrumentation: cadaver lab, World neurosurgery, 126,e1449-e1455, http://doi.org/10.1016/j.wneu.2019.03.118, 2019.

[7] El-Hariri. H, Pandey. P, Hodgson. A. J, and Garbi. R, Augmented reality visualization for orthopaedic surgical guidance with pre-and intraoperative multimodal image data fusion, Healthcare Technology Letters, 5(5), pp. 189-193, http://doi.org/10.1049/htl.2018.5061, 2018.

[8] Vávra. P, Roman. J, Zonča. P, Ihnát. P, Němec. M, Kumar. J, and ElGendi. A, Recent development of augmented reality in surgery: a review, Journal of healthcare engineering, 2017.

[9] Kim. S. K, Kang. S. J, Choi. Y. J, Choi. M. H, and Hong. M, Augmented-reality survey: from concept to application, KSII Transactions on Internet and Information Systems (TIIS), 11(2), pp. 982-1004, https://doi.org/10.3837/tiis.2017.02.019, 2017.
[10] Frajhof. L, Borges. J, Hoffmann. E, Lopes. J, and Haddad. R, Virtual reality, mixed reality and augmented reality in surgical planning for video or robotically assisted thoracoscopic anatomic resections for treatment of lung cancer, Journal of Visualized Surgery, 4, pp. 143, https://doi.org/10.21037/jovs.2018.06.02, 2018.

[11] Mahmood. F, Mahmood. E, Dorfman. R. G, Mitchell. J, Mahmood. F. U, Jones. S. B, and Matyal. R, Augmented reality and ultrasound education: initial experience, Journal of cardiothoracic and vascular anesthesia, 32(3), pp. 1363-1367, https://doi.org/10.1053/j.jvca.2017.12.006, 2018.

[12] Wilhelm. D, Vogel. T, Ostler. D, Marahrens. N, Kohn. N, Koller. $\mathrm{S}$, and Kranzfelder. M, Enhanced visualization: from intraoperative tissue differentiation to augmented reality, Visceral medicine, 34(1), pp.52-59, https://doi.org/10.1159/000485940, 2018.

[13] Liu. Y, and Tang. P, The prospect for the application of the surgical navigation system based on artificial intelligence and augmented reality, In 2018 IEEE international conference on artificial intelligence and virtual reality (AIVR), pp. 244-246, IEEE, https://doi.org/10.1109/AIVR.2018.00056, 2018.

[14] Jones. L. D, Golan. D, Hanna. S. A, and Ramachandran. M, Artificial intelligence, machine learning and the evolution of healthcare: A bright future or cause for concern?, Bone \& joint research, 7(3), pp. 223- 225, https://doi.org/10.1302/2046-3758.73.BJR-2017-0147.R1, 2018.

[15] Chen. M, Ling. C, and Zhang. W, Analysis of augmented reality application based on cloud computing, In 2011 4th International Congress on Image and Signal Processing, Vol. 2, pp. 569-572, IEEE, https://doi.org/10.1109/CISP.2011.6100311, 2011.

[16] Ahad. M. A. R, Kobashi. S, and Tavares. J. M. R, Advancements of image processing and vision in healthcare, Journal of Healthcare Engineering, pp.3-6, https://doi.org/10.1155/2018/8458024, 2018.

[17] Pereira. N, Kufeke. M, Parada. L, Troncoso. E, Bahamondes. J, Sanchez. L, and Roa. R, Augmented reality microsurgical planning with a smartphone (ARM-PS): a dissection route map in your pocket, Journal of Plastic, Reconstructive \& Aesthetic Surgery, 72(5), pp. 759762, https://doi.org/10.1016/j.bjps.2018.12.023, 2019.

[18] Khor. W. S, Baker. B, Amin. K, Chan. A, Patel. K, and Wong. J, Augmented and virtual reality in surgery - the digital surgical environment: applications, limitations and legal pitfalls, Annals of translational medicine, 4(23), https://doi.org/10.21037/atm.2016.12.23, 2016.

[19] Pereira-Azevedo. N. M, and Venderbos. L. D, eHealth and mHealth in prostate cancer detection and active surveillance, Translational andrology and urology, 7(1), pp. 170, https://doi.org/10.21037/tau.2017.12.22, 2018.

[20] Viglialoro. R. M, Condino. S, Turini. G, Carbone. M, Ferrari. V, and Gesi. M, Review of the augmented reality systems for shoulder rehabilitation, Information, 10(5), pp. 154, https://doi.org/10.3390/info10050154, 2019.

[21] Garrett. B. M, Anthony. J, and Jackson. C, Using mobile augmented reality to enhance health professional practice education, Current Issues in Emerging eLearning, 4(1), pp. 10, https://scholarworks.umb.edu/ciee/vol4/iss1/10, 2018.

[22] Wong. K, Yee. H. M, Xavier. B. A, and Grillone. G. A, Applications of augmented reality in otolaryngology: a systematic review, Otolaryngology-Head and Neck Surgery, 159(6), pp. 956-967, https://doi.org/10.1177/0194599818796476, 2018.

[23] Yoon. J. W, Chen. R. E, Kim. E. J, Akinduro. O. O, Kerezoudis. P, Han. P. K, and Quinones-Hinojosa. A, Augmented reality for the surgeon: systematic review, The International Journal of Medical Robotics and Computer Assisted Surgery, 14(4), e1914, https://doi.org/10.1002/rcs.1914, 2018.

[24] Belmustakov. S, Bailey. C, and Weiss. C. R, Augmented and virtual reality navigation for interventions in the musculoskeletal system, Current Radiology Reports, 6(9), pp. 1-10, https://doi.org/10.1007/s40134-0180293-5, 2018

[25] Uppot. R. N, Laguna. B, McCarthy. C. J, De Novi. G, Phelps. A, Siegel. E, and Courtier. J, Implementing virtual and augmented reality tools for radiology education and training, communication, and clinical care, Radiology, 291(3), pp. 570-580, https://doi.org/10.1148/radiol.2019182210, 2019.

[26] Rabbi. I, Ullah. S, and Khan. S. U, Augmented Reality Tracking Techniques: A Systematic Literature Review Protocol, IOSR Journal of Computer Engineering (IOSRJCE) ISSN, pp. 2278-0661, https://doi.org/10.9790/0661-0222329, 2012 
[27] Pflugi. S, Lerch. T, Vasireddy. R, Boemke. N, Tannast. M, Ecker. T. M, and Zheng. G, Augmented Marker Tracking for Periacetabular Osteotomy Surgery: A Cadaver Study, CAOS, 1, pp. 54-57, https://doi.org/10.29007/9mbb, 2017.

[28] García-Cruz. E, Bretonnet. A, and Alcaraz. A, Testing smart glasses in urology: clinical and surgical potential applications, Actas Urológicas Españolas, 42(3), pp. 207-211, https://doi.org/10.1016/j.acuroe.2018.02.004, 2018.

[29] Nakamoto. M, Ukimura. O, Faber. K, and Gill. I. S, Current progress on augmented reality visualization in endoscopic surgery, Current opinion in urology, 22(2), pp. 121-126, https://doi.org/10.1097/MOU.0b013e3283501774, 2012.

[30] Daher. S, Optical see-through vs. spatial augmented reality simulators for medical applications, In 2017 IEEE Virtual Reality (VR), pp. 417418, IEEE.https://doi.org/10.1109/VR.2017.7892354, 2017.

[31] Hemanth. J. D, Kose. U, Deperlioglu. O, and de Albuquerque. V. H. C, An augmented reality- supported mobile application for diagnosis of heart diseases, The Journal of Supercomputing, 76(2), pp. 1242-1267, https://doi.org/10.1007/s11227-018-2483-6, 2020.

[32] Moro. C, Štromberga. Z, Raikos. A, and Stirling. A, The effectiveness of virtual and augmented reality in health sciences and medical anatomy, Anatomical sciences education, 10(6), pp. 549-559, https://doi.org/10.1002/ase.1696, 2017.

[33] Lahanas. V, Georgiou. E, and Loukas. C, Surgical simulation training systems: box trainers, virtual reality and augmented reality simulators, International Journal of Advanced Robotics and Automation, 1(2), pp. 1-9, https://doi.org/10.15226/2473-3032/1/2/00109, 2016.

[34] Maier-Hein. L, Mountney. P, Bartoli. A, Elhawary. H, Elson. D, Groch. A, and Stoyanov. D, Optical techniques for 3D surface reconstruction in computer-assisted laparoscopic surgery, Medical image analysis, 17(8), pp. 974-996, https://doi.org/10.1016/j.media.2013.04.003, 2013.

[35] Chen. P. H, Gadepalli. K, MacDonald. R, Liu. Y, Nagpal. K, Kohlberger. $\mathrm{T}$, and Stumpe. M. C, An augmented reality microscope for realtime automated detection of cancer, In Proc. Annu. Meeting American Association Cancer Research, 2018.

[36] Tabrizi. L. B, and Mahvash. M, Augmented reality-guided neurosurgery: accuracy and intraoperative application of an image projection technique, Journal of neurosurgery, 123(1), pp. 206-211, 2015.
[37] Cavalcanti. V. C, Santana. M. I. D, Da Gama. A. E, and Correia. W. F, Usability assessments for augmented reality motor rehabilitation solutions: a systematic review, International Journal of Computer Games Technology, 2018, https://doi.org/10.1155/2018/5387896, 2018.

[38] Hussain. R, Lalande. A, Marroquin. R, Girum. K. B, Guigou. C, and Grayeli. A. B, Real- time augmented reality for ear surgery, In International Conference on Medical Image Computing and Computer-Assisted Intervention, pp. 324-331, Springer, Cham, https://doi.org/10.1007/9783-030 00937-3_38, 2018.

[39] Monge. J, and Postolache. O, Augmented reality and smart sensors for physical rehabilitation, In 2018 International Conference and Exposition on Electrical And Power Engineering (EPE), pp. 1010-1014, IEEE.https://doi.org/10.1109/ICEPE.2018.8559935, 2018.

[40] Hsiao. K. F, and Rashvand. H. F, Data modeling mobile augmented reality: integrated mind and body rehabilitation, Multimedia Tools and Applications, 74(10), pp. 3543-3560, https://doi.org/10.1007/s11042013-1649-8, 2015.

[41] Shinomiya. A, Shindo. A, Kawanishi. M, Miyake. K, Nakamura. T, Matsubara. S, and Tamiya. T, Usefulness of the 3D virtual visualization surgical planning simulation and 3D model for endoscopic endonasal transsphenoidal surgery of pituitary adenoma: technical report and review of literature, Interdisciplinary Neurosurgery, 13, pp. 13-19, https://doi.org/10.1016/j.inat.2018.02.002, 2018.

[42] Gallos. P, Georgiadis. C, Liaskos. J, and Mantas. J, Augmented Reality Glasses and Head-Mounted Display Devices in Healthcare, Studies in health technology and informatics, 251, pp. 82-85, 2018.

[43] Ingeson. M, Blusi. M, and Nieves. J. C, Smart augmented reality mHealth for medication adherence, In AIH@ IJCAI, pp. 157-168, 2018.

[44] Chamberlain. D., Jimenez-Galindo. A, Fletcher. R. R., and Kodgule. $\mathrm{R}$, Applying augmented reality to enable automated and low-cost data capture from medical devices, In Proceedings of the Eighth International Conference on Information and Communication Technologies and Development, pp. 1-4, https://doi.org/10.1145/2909609.2909626, 2016.

[45] Hayhurst. J, How augmented reality and virtual reality is being used to support people living with dementia-Design challenges and future directions, Augmented reality and virtual reality, pp. 295-305, https://doi.org/10.1007/978-3-319-64027-3_20, 2018. 